\title{
Characterization of common carp (Cyprinus carpio L.) interferon regulatory factor 5 (IRF5) and its expression in response to viral and bacterial challenges
}

\author{
Yaoyao Zhu, Chenchen Qi, Shijuan Shan, Fumiao Zhang, Hua Li, Liguo An* and Guiwen Yang
}

\begin{abstract}
Background: Common carp (Cyprinus carpio L.), one of the most economically valuable commercial farming fish species in China, is often infected by a variety of viruses. As the first line of defence against microbial pathogens, the innate immune system plays a crucial role in teleost fish, which are lower vertebrates. Interferon (IFN) regulatory factor 5 (IRF5) is a key molecule in antiviral immunity that regulating the expression of IFN and other pro-inflammatory cytokines. It is necessary to gain more insight into the common carp IFN system and the function of fish IRF5 in the antiviral and antibacterial response.

Results: In the present study, we characterized the cDNA and genomic sequence of the IRF5 gene in common carp, and analysed tissue distribution and expression profile of this gene in response to polyinosinic:polycytidylic acid (poly l: C) and lipopolysaccharides (LPS) treatment. The common carp IRF5 (cCIRF5) gene is 5790 bp in length and is composed of 9 exons and 8 introns. The open reading frame (ORF) of cclRF5 is $1554 \mathrm{bp}$, and encodes 517 amino acid protein. The putative cClRF5 protein shares identity (65.4-90.0 \%) with other fish IRF5s and contains a DNA binding domain (DBD), a middle region (MR), an IRF-associated domain (IAD), a virus activated domain (VAD) and two nuclear localization signals (NLSs) similar to those found in vertebrate IRF5. Phylogenetic analysis clustered ccIRF5 into the IRF5 subfamily with other vertebrate IRF5 and IRF6 genes. Real-time PCR analysis revealed that ccIRF5 mRNA was expressed in all examined tissues of healthy carps, with high levels observed in the gills and the brain. After poly I:C challenge, expression levels of cCIRF5, tumour-necrosis factor a (ccTNFa) and two IFN stimulated genes [ISGs (ccISG5 and ccPKR)] were up-regulated in seven immune-related tissues (liver, spleen, head kidney, foregut, hindgut, skin and gills). Furthermore, all four genes were up-regulated in vitro upon poly I:C and LPS challenges.
\end{abstract}

Conclusions: Our findings suggest that IRF5 might play an important role in regulating the antiviral and antibacterial response in fish. These results could provide a clue for preventing common carp infection by pathogenic microorganisms present in the aquatic environment.

Keywords: IRF5, Common carp (Cyprinus carpio L.), Poly I:C, LPS, Gene expression

\footnotetext{
*Correspondence: an.liguo58@gmail.com; yanggw@sdnu.edu.cn

Shandong Provincial Key Laboratory of Animal Resistance Biology, College of

Life Science, Shandong Normal University, No. 88 East Wenhua Road, Jinan

250014, People's Republic of China
} 


\section{Background}

Interferon (IFN) regulatory factors (IRFs) are key transcriptional mediators of virus-, bacteria- and IFNinduced signalling pathways, and they play an important role in antiviral defence, immune response, cell growth regulation, apoptosis and oncogenesis [1-3]. IRFs were originally identified as participating in the transcriptional regulators of IFN and IFN stimulated genes (ISGs) [4]. To date, eleven IRF family members have been identified in vertebrate, including IRF1, IRF2, IRF3, IRF4 [also known as PU.1 interaction partner (PIP), lymphoidspecific IRF (LSIRF) or consensus sequence-binding protein in adult T-cell leukemia cell line or activated T cells (ICSAT)], IRF5, IRF6, IRF7, IRF8 [also known as IFN consensus sequence binding protein (ICSBP)], IRF9 [also called as ISG factor 3 gamma (ISGF3 $\gamma$ )], IRF10 and IRF11. However, IRF10 and 11 have been identified in fish but in mammalians [5]. IRFs used to be classified into three groups according to differences in the $\mathrm{C}$ terminal region: activators (IRF1, 3, 5, 7, 9 and 10), repressors (IRF2 and 8) and multifunctional factors that both activate and repress gene transcription (IRF2, 4, 5 and 7) [3, 4]. All members share a well-conserved Nterminal DNA-binding domain (DBD), and the conserved tryptophan repeat cluster in the first 120 amino acids of the DBD is responsible for binding the promoters of target genes [6]. IRF-associated domain (IAD) in the C-terminal of IRF3-11 mediates the interactions between IRFs and other protein to form transcriptional complexes [7].

Human IRF5 plays a crucial role in regulating the expression of IFN- $\alpha$ and IFN- $\beta$, mediating the virus- and cell type-specific immune response. IRF5 is also critical for the retinoic acid-inducible gene I (RIG-I) and Tolllike receptors immune pathways [8-12]. Other reports have also indicated that IRF5 may promote lymphocyte differentiation and apoptosis [9]. IRF5 knockout mice were reported to be susceptible to viral infections, while, the expression levels of type I IFNs and other proinflammatory cytokines including tumour-necrosis factor (TNF)- $\alpha$, interleukin (IL)-6 and IL-12, were reduced in response to viral infections $[12,13]$.

IRF5 have been previously identified in several fish species, including grass carp (Ctenopharyngodon idella) [14], zebrafish (Danio rerio) [15], Atlantic salmon (Salmo salar) [16], Japanese flounder (Paralichthys olivaceus) [17], rock bream (Oplegnathus fasciatus) [18], halfsmooth tongue sole (Cynoglossus semilaevis) [19], channel catfish (Ictalurus Punctatus) [20], Mi-iuy croaker (Miichthys miiuy) (unpublished data) and paddlefish (Polyodon spathula) [21]. Common carp (Cyprinus carpio L.), one of the most economically valuable commercial farming fish species, is often infected by a wide variety of viruses $[22,23]$. To date, among the IRFs, only IRF3 and
IRF7 have been identified in common carp [22]. Additional studies are needed to gain a better insight into the IFN system in common carp and the function of fish IRF5 in the antiviral and antibacterial immune response. In the present study, we characterized the cDNA sequence and genomic structure of IRF5 from common carp. We reported the evolutionary relationship of common carp IRF5 (ccIRF5) gene with other IRF genes. What's more, we analysed its tissue-specific distribution in twelve tissues and expression profiles upon polyinosinic:polycytidylic acid (poly I:C) and lipopolysaccharides (LPS) stimulation both in vivo and in vitro by using real-time PCR.

\section{Methods}

\section{Fish and poly I:C challenge}

Common carp (body weight approximately 200 g) were obtained from the Fresh Water Fishery Research Institute of Shandong Province, China. Fish were reared in aerated freshwater tanks at $24-26^{\circ} \mathrm{C}$ and were fed twice per day with commercial carp pellets (Shandong Tianshen Fishing Feedstuff Co., Ltd). After acclimatization for 1 week, the fish were used for the experiments.

Poly I:C (Sigma, USA) was re-suspended in phosphatebuffered saline (PBS) for the immune challenge experiments. Fish were intraperitoneally injected with $500 \mu \mathrm{l}$ of a poly I:C solution $(2.6 \mathrm{mg} / \mathrm{ml})$ per fish, while un-challenged fish served as controls (indicated as $0 \mathrm{~h}$ ). After injection, all fish were placed into a rectangular tank containing fresh water. At $0,3,6,12,24,48$ and $72 \mathrm{~h}$ post-injection (hpi), three fish in each group were euthanized and seven tissues including liver, spleen, head kidney, skin, gills, foregut and hindgut were sampled for total RNA extraction.

\section{RNA and genomic DNA extraction}

Total RNA was extracted from different tissues of common carp using an RNA simple Total RNA Kit (TIANGEN) according to the manufacturer's instructions. Total RNA quality and concentration were determined by $1 \%$ gel electrophoresis and ultraviolet spectrophotometry, respectively. RNA samples had an OD260:OD280 ratio between 1.8 and 2.0. Genomic DNA was then removed and first-strand cDNA was synthesized by using the FastQuant RT Kit (containing gDNase) (TIANGEN) following the manufacturer's protocol. Genomic DNA was extracted from the spleen of healthy fish using a TIANamp Genomic DNA Kit (TIANGEN).

\section{Cloning of cDNA and genomic sequences}

A pair of primers, IRF5-F and IRF5-R (shown in Table 1), designed based on the conserved regions of different IRF5 genes, was used to amplify the corresponding ccIRF5 sequence from spleen cDNA of healthy fish. The PCR cycling parameters were $94{ }^{\circ} \mathrm{C}$ for $5 \mathrm{~min}$, followed by 30 cycles of $94{ }^{\circ} \mathrm{C}$ for $30 \mathrm{~s}, 54{ }^{\circ} \mathrm{C}$ for $30 \mathrm{~s}, 72{ }^{\circ} \mathrm{C}$ for 
Table 1 Primers used in this study

\begin{tabular}{|c|c|c|c|}
\hline Primer & Sequence $\left(5^{\prime}-3^{\prime}\right)$ & Application & GenBank No. \\
\hline $\begin{array}{l}\text { IRF5-F } \\
\text { IRF5-R }\end{array}$ & $\begin{array}{l}\text { GCAAGTACCAGGAAGGAG } \\
\text { AAATCAGTTAAGGGCAACA }\end{array}$ & Homologous amplification & \\
\hline $\begin{array}{l}\text { IRF5-3 F1 } \\
\text { IRF5-3 F2 } \\
\text { IRF5-5R1 } \\
\text { IRF5-5R2 } \\
\text { IRF5g1-F } \\
\text { IRF5g1-R }\end{array}$ & $\begin{array}{l}\text { GTCACTCATTCTGTAGGCCCT } \\
\text { GCAGAGAACCAGGTTCATCC } \\
\text { TGTCCTTCGTCCATCGTAGTG } \\
\text { GTTAGGGCACAGCGGAGATT } \\
\text { CAGCACCATGAGTGGTCAACCACG } \\
\text { AATATAGAGTTCTCCTCCTCCAG }\end{array}$ & $\begin{array}{l}\text { First round 3'-RACE PCR } \\
\text { Nested 3'-RACE PCR } \\
\text { First round 5'-RACE PCR } \\
\text { Nested 5'-RACE PCR } \\
\text { For the first intron }\end{array}$ & \\
\hline $\begin{array}{l}\text { IRF5g2-F } \\
\text { IRF5g2-R }\end{array}$ & $\begin{array}{l}\text { AGGACTGCACTGGCTCAATCAAGA } \\
\text { ATCTACTCCTTCCTGATACTTGCCT }\end{array}$ & For the second intron & \\
\hline $\begin{array}{l}\text { IRF5g3-F } \\
\text { IRF5g3-R }\end{array}$ & $\begin{array}{l}\text { ATGGAAAGCCAATCTCCGCTGTG } \\
\text { GTAACATGAGCGGACTGCTCAGC }\end{array}$ & For the third, fourth and fifth intron & \\
\hline $\begin{array}{l}\text { IRF5g4-F } \\
\text { IRF5g4-R }\end{array}$ & $\begin{array}{l}\text { AGTGCAATCAAAGTTGAGCAGGCA } \\
\text { ATGATCGGTATAGAACCTCTGCTT }\end{array}$ & For the sixth intron & \\
\hline $\begin{array}{l}\text { IRF5g5-F } \\
\text { IRF5g5-R }\end{array}$ & $\begin{array}{l}\text { ACGCCATCAGGCTGTGCCAGTGTA } \\
\text { CAATGATGAGCTITITCTCCTTG }\end{array}$ & For the seventh intron & \\
\hline $\begin{array}{l}\text { IRF5g6-F } \\
\text { IRF5g6-R }\end{array}$ & $\begin{array}{l}\text { GCTTCGGTGAGGACTGGCCAGACA } \\
\text { ACTCTGCAGAAGTCTGTGGAGCT }\end{array}$ & For the eighth intron & \\
\hline $\begin{array}{l}\text { IRF5-rF } \\
\text { IRF5-rR }\end{array}$ & $\begin{array}{l}\text { CCTGGGCTCAGAACATCCACTAAC } \\
\text { AGTATGGCATCATAGAGGGCACCT }\end{array}$ & Real-time PCR & \\
\hline $\begin{array}{l}\text { TNFa-F } \\
\text { TNFa-R }\end{array}$ & $\begin{array}{l}\text { ACAGGTGATGGTGTCGAGGAGGA } \\
\text { TCTGAGACTTGTTGAGCGTGAAG }\end{array}$ & Real-time PCR & JF957372 \\
\hline $\begin{array}{l}\text { S11-F } \\
\text { S11-R }\end{array}$ & $\begin{array}{l}\text { CCGTGGGTGACATCGTTACA } \\
\text { TCAGGACATTGAACCTCACTGTCT }\end{array}$ & Real-time PCR & AB012087 \\
\hline ISG15-F & GTGAGCGGTGAAGCCACAGTTG & Real-time PCR & KP115358 \\
\hline ISG15-R & GCGAACCGTTATCGGCAGACAG & & \\
\hline PKR-F & AGGCTTGATCCACAGAGACCTGAA & Real-time PCR & JX516101 \\
\hline PKR-R & CGTTCCAGAAGTTGCACGTCATTG & & \\
\hline
\end{tabular}

$50 \mathrm{~s}$, and a final extension step of $72{ }^{\circ} \mathrm{C}$ for $10 \mathrm{~min}$. Then, the full-length cDNA of ccIRF5 was obtained by the rapid amplification of the cDNA ends (RACE) method using a 3'-Full RACE and 5'-Full RACE Core Set Kit (TaKaRa). The detailed procedure was performed as described in the user manual, and two primers pairs (IRF5-3 F1/-3 F2 and IRF5-5R1/5R2) were used in the RACE-PCR (shown in Table 1).

PCR products were analysed by electrophoresis on a $1 \%$ agarose gel and the anticipated fragments were purified from agarose gels. These fragments were ligated into the pMD18-T vector (TaKaRa) and transformed into competent Escherichia coli DH-5 $\alpha$ competent cells, and recombinants were identified and sequenced (Invitrogen).

\section{Sequence and phylogenetic analysis}

The full-length cDNA sequence of ccIRF5 was compared with other corresponding IRF5 sequences by using the BLAST program from the National Center for Biotechnology Information (NCBI) [24] and the MegAlign program DNASTAR. The putative ORF and the protein prediction were analysed with Editseq within DNASTAR. The protein domains were predicted with simple modular architecture research tool (SMART) [25].
Phylogenetic analysis was performed with MEGA 5.0, using the neighbor-joining method. Homologous sequences were searched using the NCBI BLAST server.

\section{Leukocyte isolation and in vitro challenges}

The methods for isolating common carp leukocytes from peripheral blood (PBLs) and head kidney (HKLs) have been described by Rymuszka et al. [26]. Peripheral blood was diluted 1:1 with Leibovitz's L-15 medium (supplemented with $10 \mathrm{U} / \mathrm{ml}$ of heparin, $10 \mathrm{mM}$ HEPES, $60 \mathrm{mM}$ $\mathrm{NaCl}, 5 \% \mathrm{FBS}, 100 \mathrm{U} / \mathrm{ml}$ penicillin, $100 \mu \mathrm{g} / \mathrm{ml}$ streptomycin, and $250 \mathrm{ng} / \mathrm{ml}$ amphotericin B) and layered on a $65 \%$ Percoll (Sigma) solution, and centrifuged at $2500 \mathrm{rpm}$ for $30 \mathrm{~min}$ at $4{ }^{\circ} \mathrm{C}$. The cells were washed by centrifugation in PBS and re-suspended in cold Leibovitz's L-15 medium.

Head kidney cell suspensions were obtained by gently pressing the tissue with a plunger through a $100-\mu \mathrm{m}$ sterile nylon mesh with Leibovitz's L-15 medium. Percoll layers of 51 and $34 \%$ were used and centrifuged at $1500 \mathrm{rpm}$ for $30 \mathrm{~min}$ at $4{ }^{\circ} \mathrm{C}$. The cell layers at the interphase were collected and washed twice with Leibovitz's L-15 medium. 
For stimulation experiments, $1 \times 10^{6}$ cells were maintained at $25{ }^{\circ} \mathrm{C}$ in a 24-well tissue culture plate with $0.5 \mathrm{mg} / \mathrm{ml}$ poly I:C and $1 \mathrm{mg} / \mathrm{ml} \mathrm{LPS} \mathrm{(suspended} \mathrm{in} \mathrm{PBS),}$ while un-challenged cells served as controls (indicated as $0 \mathrm{~h}$ ). The cells were harvested at 0, 3, 6, 9, 12 and $24 \mathrm{hpi}$ and RNA was extracted and reverse transcribed.

\section{Real-time quantitative PCR}

The inducible expression profiles of ccIRF5, ccTNFa and two ISG genes [ccISG15 and ccPKR (protein kinase R)] upon poly I:C and LPS challenges were analysed by realtime PCR using gene-specific primers (Table 1) on an iQ5 Real-time PCR instrument (Bio-Rad). Reactions were performed in a $20 \mu \mathrm{l}$ volume using SYBR Green RealMasterMix (TIANGEN). Cycling conditions were one cycle of $94{ }^{\circ} \mathrm{C}$ for $5 \mathrm{~min}$, followed by 40 cycles of $94{ }^{\circ} \mathrm{C}$ for $20 \mathrm{~s}$, $60{ }^{\circ} \mathrm{C}$ for $30 \mathrm{~s}$ and $72{ }^{\circ} \mathrm{C}$ for $20 \mathrm{~s}$. The $40 \mathrm{~S}$ ribosomal protein $\mathrm{S} 11$ gene was amplified in parallel as a housekeeping control for normalization [27]. The amplification efficiency of the primers used in the real-time PCR was between 0.80 and 0.86. All samples were analysed in triplicate. Standard curves were run on the same plate. The real-time PCR data were analysed with the $2^{-\triangle \Delta C T}$ method.

\section{Statistical analysis}

Differences in relative gene expression between the challenged group and the control group were tested using one-way analysis of variance (ANOVA) in Graphpad Prism 5. $P$ values $<0.05$ were considered statistically significant.

\section{Results}

Characterization of the CDNA sequence of ccIRF5

The full-length cDNA of ccIRF5 (GenBank Accession No.: KP979609) was 2042 bp, consisting of a 5'- untranslated region (UTR) of $266 \mathrm{bp}$, a 3'-UTR of 222 bp with a polyadenylation signal (AATAAA) starting $30 \mathrm{bp}$ upstream of the poly (A) tail and an ORF of 1554 bp encoding a peptide of 517 amino acids. The predicted molecular weight of ccIRF5 is $58.5 \mathrm{kDa}$, and the isoelectric point is 5.8. The deduced amino acid sequence of ccIRF5 shares higher identities with the fish IRF5s (65.4 \% of P. spathula to $90.0 \%$ of C. idella) than with IRF5s of other species (51.6\% of G. gallus to $56.7 \%$ of M. musculus) (Table 2) and contains a DBD, a middle region (MR), an IAD and a virus activated domain (VAD) (Fig. 1).

\section{Genomic structure of cclRF5}

The ccIRF5 gene is 5798 bp and is composed of 9 exons and 8 introns (Fig. 2). The exon-intron splice junctions follow the AG/GT rule (Table 3). The ccIRF5 gene has the same size exons as the grass carp IRF5, while, the introns sizes are different, which are 495, 804, 225, 131, 88, 761, 518, and 766 bp (listed in Additional file 1).

\section{Phylogenetic analysis}

The phylogenetic tree was constructed based on a Clustal W alignment using MEGA 5.0 by the neighbor-joining method. All IRF members were divided into four subfamilies: IRF1, IRF2, and zebrafish IRF11 belonged to the IRF1 subfamily, IRF3 and IRF7 belonging to the IRF3 subfamily; IRF4, 8, 9, and 10 belonging to the IRF4 subfamily; IRF5 and IRF6 belonging to the IRF5 subfamily. In the phylogenetic tree, ccIRF5 showed a closer relationship with genes from other cyprinid fish, including grass carp and zebrafish IRF5s, and clustered into the fish IRF5 subgroup (Fig. 3).

Table 2 Amino acid identities (\%) of ccIRF5 to other vertebrate IRF5 proteins

\begin{tabular}{lllll}
\hline Species & Protein length (aa) & GeneBank No. & Full-length identities & DBD identities \\
\hline Ctenopharyngodon idella & 519 & ACT83675 & 90.0 & 87.9 \\
Danio rerio & 498 & ABY91289 & 76.8 & 93.5 \\
Ictalurus Punctatus & 478 & AHH37262 & 68.9 & 85.1 \\
Paralichthys olivaceus & 472 & AEY55357 & 69.7 & 75.4 \\
Scophthalmus maximus & 487 & AEG76960 & 69.6 & 75.4 \\
Salmo salar & 532 & NP_001133324 & 69.7 & 82.5 \\
Oplegnathus fasciatus & 498 & AFZ93894 & 70.1 & 80.7 \\
Miichthys miiuy & 492 & AHB59743 & 65.4 & 80.7 \\
Polyodon spathula & AEW27153 & 56.9 & 74.6 \\
Xenopus laevis & 496 & NP_001088065 & 51.6 & 73.7 \\
Gallus gallus & 517 & NP_001026758 & 58.8 \\
Mus musculus & 472 & AAB81997 & 56.7 \\
Homo sapiens & 497 & AAH04201 & 55.3 \\
\hline
\end{tabular}




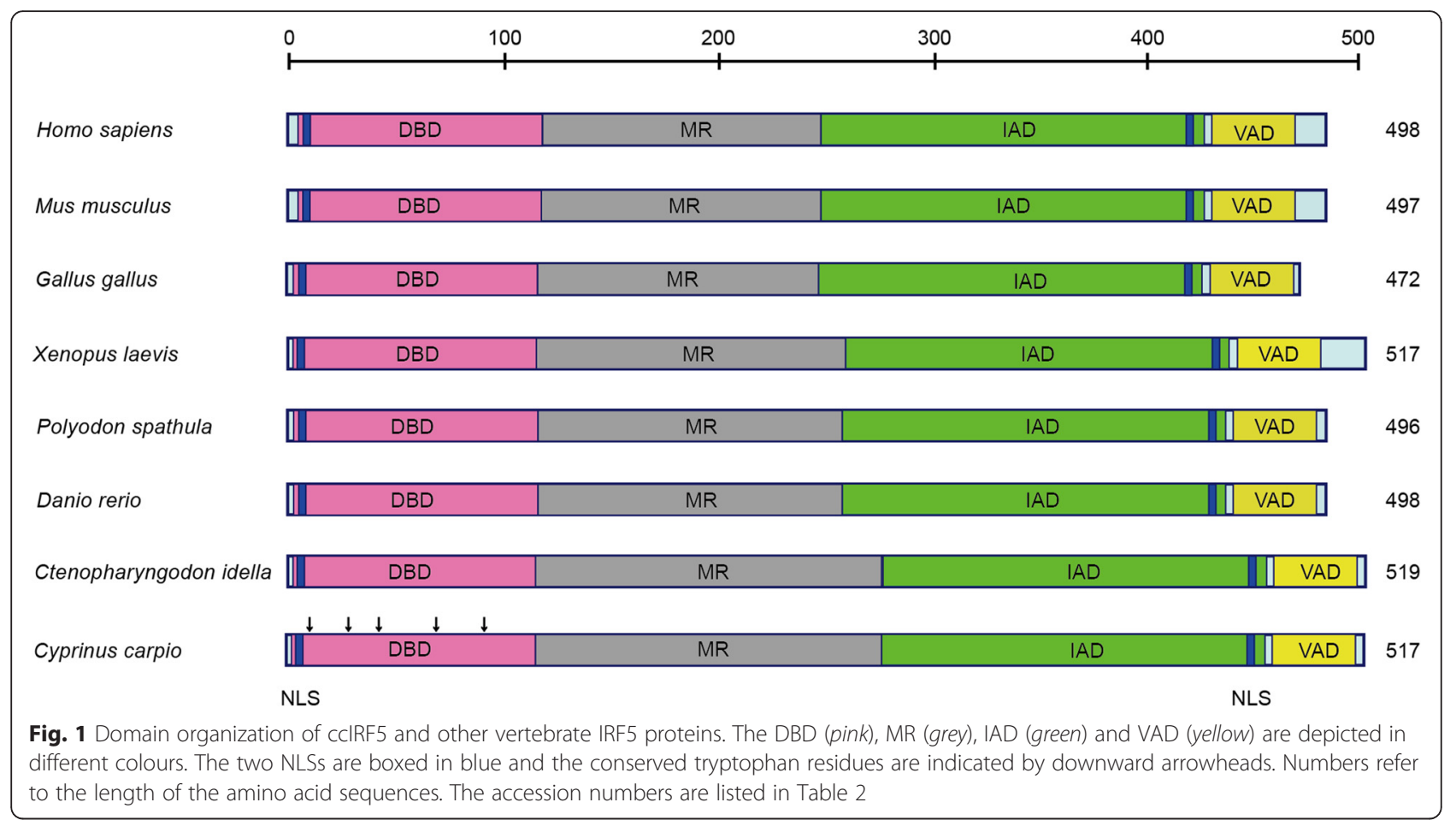

Tissue distribution of ccIRF5 mRNA in healthy carps

Constitutive expression of ccIRF5 mRNA in various tissues (gills, brain, skin, spleen, gonad, head kidney, buccal epithelium, hindgut, foregut, muscle, liver and blood) of healthy carps was examined by real-time PCR.
The highest expression levels were in the gills and the brain, followed by the skin, the spleen, the gonad, the head kidney, the buccal epithelium, the hindgut, the foregut and the muscle, while low expression was observed in the liver and the blood (Fig. 4).

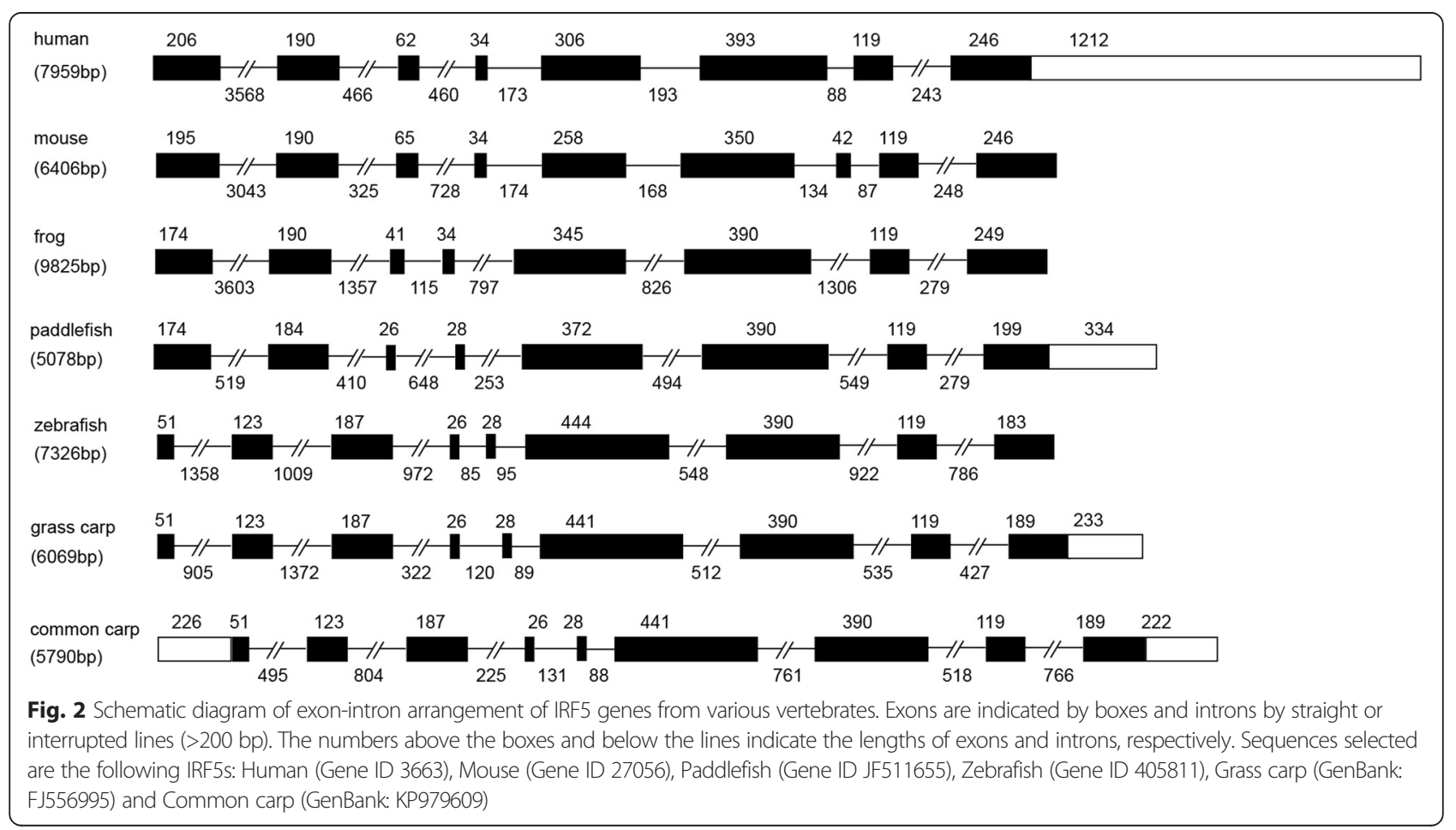


Table 3 Intron-exon junctions and flanking sequences of the ccIRF5 gene

\begin{tabular}{llllll}
\hline Exon no. & Position in DNA & Exon size $(\mathrm{bp})$ & Splice donor & Splice acceptor & Intron size $(\mathrm{bp})$ \\
\hline 1 & $1-277$ & 277 & gtgaattgtg & accctgccag & 495 \\
2 & $773-896$ & 123 & gtgagtaata & tgctcttcag & 804 \\
3 & $1701-1888$ & 187 & gtaaggaccg & ttgattttag & 225 \\
4 & $2114-2140$ & 26 & gtaagttctg & tgcctttcag & 131 \\
5 & $2272-2300$ & 28 & gtaagatttt & tgcttgacag & 88 \\
6 & $2389-2830$ & 441 & gtaagcctct & tcttctctag & 761 \\
7 & $3592-3982$ & 390 & gtaagaggaa & ttcttctcag & 518 \\
8 & $4501-4620$ & 119 & gtttggagat & cgttatctag & 766 \\
\hline
\end{tabular}

Bold text indicates the invariant nucleotides of the exon-intron boundaries

\section{Gene expression of ccIRF5 in response to poly I:C challenge in vivo}

Temporal expression of ccIRF5 upon poly I:C challenge in seven immune-related tissues (liver, spleen, head kidney, foregut, hindgut, skin and gills) was examined at 0 , $3,6,12,24,48$ and 72 hpi using real-time PCR. The highest expression levels in different tissues were reached at different time points. The maximum induction of ccIRF5 in mucosaassociated tissues including the foregut, the hindgut, the skin and the gills, occurred within $24 \mathrm{hpi}$. In contrast, the expression levels in the spleen, the head kidney and the liver were up-regulated at later time points (48 and $72 \mathrm{hpi}$ ). The expression of ccIRF5 was weakly up-regulated in all tested tissues $(1.7$ - to 4.2 -fold, $p<0.01$ or $p<0.05)$ except in the foregut (22.8-fold increase, $p<0.01$ ) (Fig. 5).

\section{Gene expression of ccTNFa and ccISGs in response to poly $\mathrm{I}: \mathrm{C}$ challenge in vivo}

The expression levels of ccTNF $\alpha$, ccISG15 and ccPKR in all tested tissues were increased at different time points upon stimulation with poly I:C. Subsequently, the expression levels decreased gradually until 48 hpi. The highest induction of ccTNF $\alpha$ was detected in the spleen (42.3- fold, $p<0.01)$ and liver (20.4-fold, $p<0.01)$, respectively. Whereas, the expression levels of ccTNF $\alpha$ in the skin (2.8-fold) and the gills (5.8-fold, $p<0.05)$ were weakly upregulated. The expression of ccISG15 and ccPKR in all tested tissues reached peak levels at 3 and 6 hpi, respectively. Fold induction of ccISG15 was significantly increased in the foregut (3464.8-fold, $p<0.05)$ and hindgut $(1364.1$ fold, $p<0.05)$. Furthermore, the highest expression of cCPKR was observed in the liver and the hindgut at $6 \mathrm{hpi}$ by 95.9 -fold and 88.1-fold, respectively (Fig. 6).

\section{Expression profiles of ccIRF5 upon poly I:C and LPS challenges in vitro}

To further investigate the antiviral and antibacterial response of ccIRF5 in vitro, common carp PBLs and HKLs were isolated and challenged them with poly I:C or LPS. In accordance with the in vivo studies, ccIRF5 transcripts in the common carp PBLs reached the peak level at 3 hpi $(2.9$-fold, $p<0.05)$ and 6 hpi $(2.0$-fold $p<$ $0.05)$, upon poly I:C and LPS challenges, respectively. As well as in the HKLs, expression of ccIRF5 reached the peak level at 24 hpi by 5.2 -fold and 3.3-fold upon poly I:C and LPS challenges, respectively (Fig. 7).

\section{Expression profiles of ccTNFa and ccISGs in poly I:C- and LPS- stimulated PBLs}

All three genes were up-regulated at $3 \mathrm{hpi}$ by both poly I:C and LPS challenges in the PBLs. However, compared to the challenge by poly I:C (4.1- to 29.1-fold), expression levels of the three genes were weakly induced upon LPS challenge (1.8- to 3.2-fold) (Fig. 8).

\section{Discussion}

The IRF family of transcription factors plays an important role in the regulation of type I IFN genes and ISGs. IRF5 genes have been identified in several vertebrates. However, there is no evidence about the identification and function of IRF5 in common carp. In the present study, we cloned the full-length cDNA and genomic sequence of ccIRF5. The deduced amino acid sequence contains a DBD, a MR, an IAD and a VAD (Fig. 1). Similar to other IRF5s in vertebrates, five tryptophan residues (W13, W28, W40, W60 and W79) are present in the DBD which forms a helix-turn-helix structure to bind to the IFN stimulated response element (IRSE)/IRF binding element (IRF-E) consensus in the target promoters [28]. The VAD that comprises all serine residues function as virus-induced phosphorylation sites. The MR which contains a proline-rich domain and is less conserved. Although this domain is also present in IRF3, 4 and 6, its function is still unclear [29]. The IAD, which was originally identified in IRF8, can form transcriptional complexes with other IRFs or transcriptional comodulators to initiate the transcription of target genes [7]. Chen W et al. reported that the dimerization 


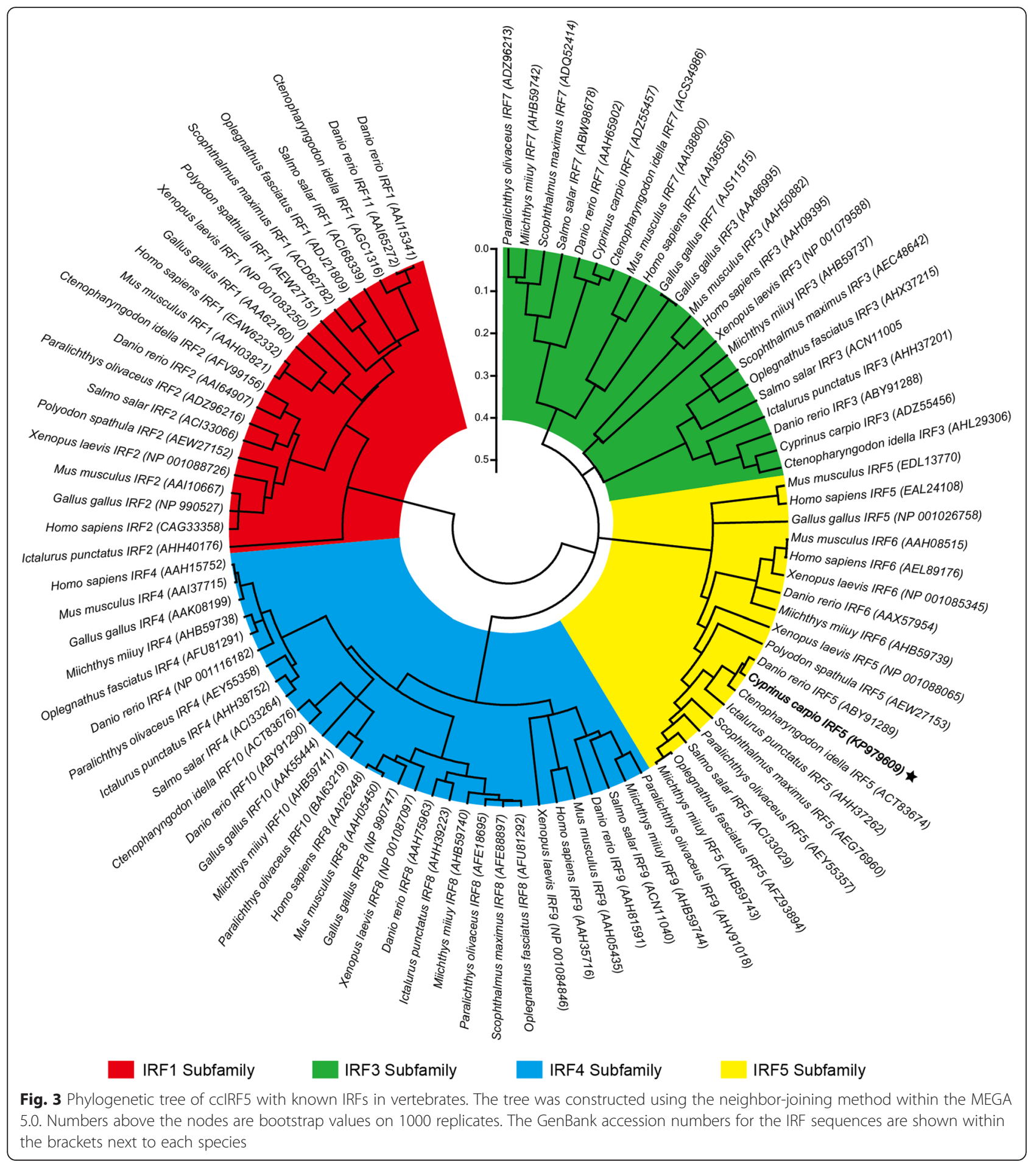

between hydrophobic and ionic interactions in the IAD played a crucial role in the high basal activity of IRFs [30]. Similar to other IRF5s, two nuclear localization signals (NLSs) are found in the $\mathrm{N}$ - and C-termini of the predicted ccIRF5 protein, and these NLRs play an important role in IRF nuclear translocation and retention in virus-infected cells $[11,14,17]$.
Similar to IRF5 genes of other Cypriniformes (zebrafish and grass carp), the genomic sequence of ccIRF5 is also composed of 9 exons and 8 introns. However, the IRF5 genes in human and several other vertebrates contain 8 exons and 7 introns. Interestingly, the sizes of the first and second exons, which encode the DBD of IRF5s in common carp, zebrafish and grass carp, are 


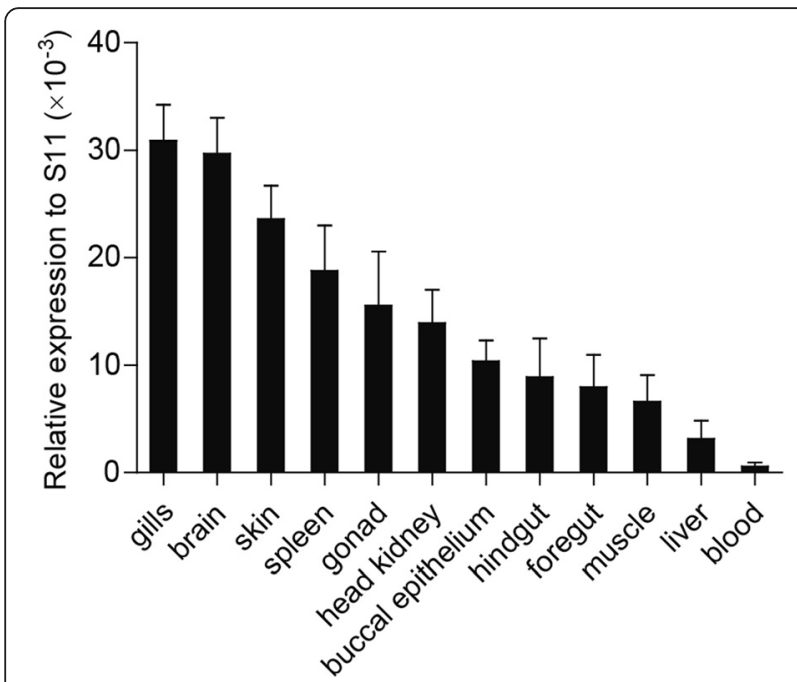

Fig. 4 Tissue distribution analysis of ccIRF5 mRNA by real-time PCR. Total RNA was isolated from various tissues of healthy carps. Gene expression levels were normalized to $40 \mathrm{~S}$ ribosomal protein S11 mRNA. The data are presented as the mean \pm S.D. $(n=3)$

similar to the size of the first exon in other vertebrates, while the other exons sizes comparable to exons in vertebrates (Fig. 2). These results suggest that the genomic structure of vertebrate IRF5s is evolutionarily conserved, and the first intron of IRF5s in common carp, zebrafish and grass carp may be lost in some other teleosts and tetrapods during evolution.

The phylogenetic tree showed that all IRF family members were divided into four subfamilies, and ccIRF5 showed a closer relationship with other cyprinids, including grass carp and zebrafish IRF5s, which were well clustered into the fish IRF5 subgroup (Fig. 3). This result matches the evolutionary relationship observed at the genomic structure level for the IRF5s in all species (Fig. 2).

The ccIRF5 transcripts were ubiquitously expressed in all tested tissues of healthy carp, which was similar to the expression of other known fish IRFs [14, 31-35]. The highest ccIRF5 expression levels in healthy common carp were in the gills and the brain, while low expression was observed in the liver and the blood (Fig. 4). Similarly, the highest expression levels of Japanese flounder and paddlefish IRF5 were also detected in the gills, which are mucosa-associated lymphoid tissues that harbour lymphocytes $[17,21]$. These results suggest that IRF5 may be crucial role for the activity of the mucosal immune system in fish. Turbot IRF5 was highly expressed in the brain, with which our result is in more agreement, suggesting that IRF5 might also play a significant role in the central nervous system [36]. High expression levels of IRF5 were shown in various tissues of different fish species. For instance, grass carp and half-smooth tongue sole IRF5s were expressed in all examined organs and the highest expression was in the spleen $[14,19]$. The rock bream IRF5 gene was highly expressed in the liver [18]. In contrast, the highest expression levels of IRF5 in zebrafish were in the ovaries and the muscle, which are not immune-associated tissues [15]. The reason for the dissimilarities in IRF5 expression patterns in different fish species may be due to the diverse immune systems of fishes.
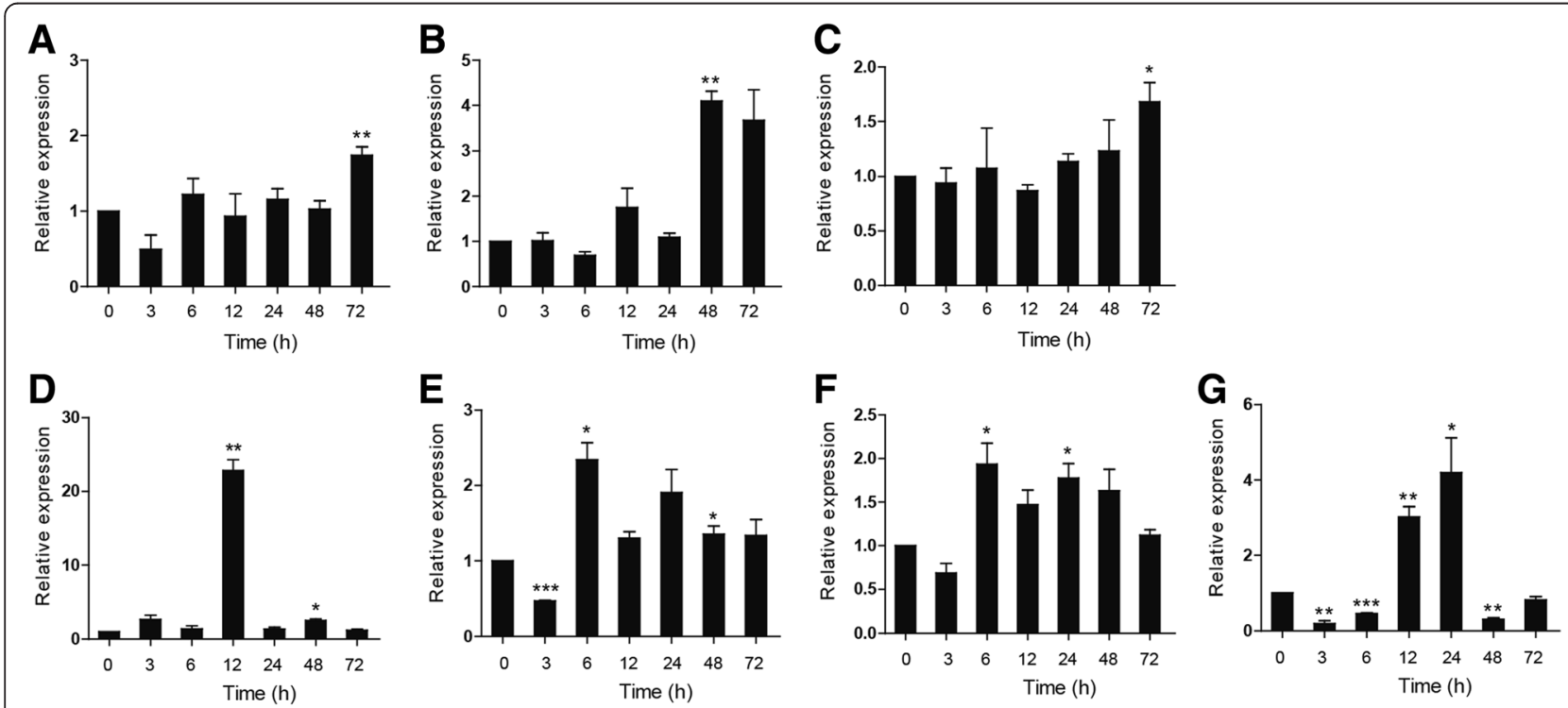

Fig. 5 Expression analysis of ccIRF5 in response to poly l:C challenge in vitro. Total RNA was extracted from head kidney (a), liver (b), spleen (c), gill (d), foregut (e), hindgut (f) and skin (g) of the control and challenged samples at 0, 3, 6, 12, 24, 48 and 72 hpi. Expression was normalized to S11 and shown as relative to control. The data are presented as the mean \pm S.D. $(n=3)$. Significant values in comparison to the control are indicated by ${ }^{*} p<0.05$, ${ }^{* *} p<0.01$ and ${ }^{* * *} p<0.001$ 

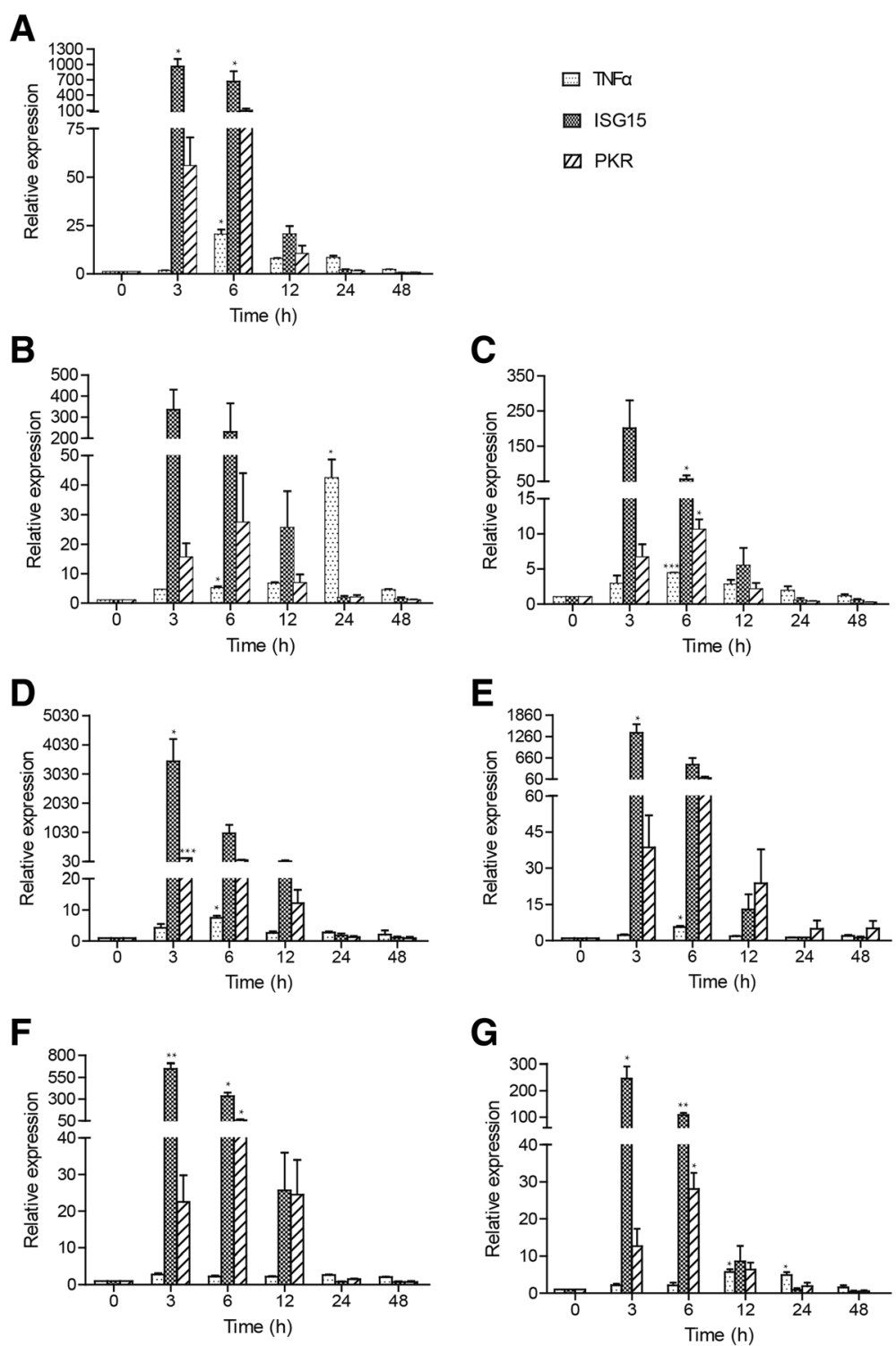

Fig. 6 Expression analysis of cCTNFa and cclSGs in response to poly l:C challenge in vitro. Total RNA was extracted from head kidney $(\mathbf{a})$, liver (b), spleen (c), gill (d), foregut (e), hindgut (f) and skin $(\mathbf{g})$ of the control and challenged samples (shown in Fig. 5). The expression was normalized to S11 and shown as relative to control. The data are presented as the mean \pm S.D. $(n=3)$. Significant values in comparison to the control are indicated by ${ }^{*} p<0.05,{ }^{* *} p<0.01$ and ${ }^{* * *} p<0.001$

Previous studies have highlighted the important role of IRF5 in regulating the expression of IFN and other proinflammatory cytokines in TLR7 and 8 antiviral responses [13]. To gain insight into the role of ccIRF5 in response to the treatment with poly I:C, which is a synthetic double-stranded RNA, the temporal expression of ccIRF5 in seven immune-related tissues was examined. As shown in Fig. 5, the expression levels of ccIRF5 in different tissues reached peak levels at different time points. The maximum induction of ccIRF5 in mucosaassociated tissues including the foregut, the hindgut, the skin and the gills, occurred within 24 hpi compared to the expression levels in the spleen, the head kidney and the liver (at 48 and $72 \mathrm{hpi}$ ). This phenomenon may be because the fish mucosal immune system is the first line of defence against the invading pathogens. The expression of ccIRF5 was slightly up-regulated in all tested tissues (1.7- to 4.2-fold), with the exception of the foregut (22.8-fold). Similarly, the expression levels of IRF3 and IRF7 mRNA were highly up-regulated in the intestine of spring viraemia of carp virus (SVCV)-infected common carps [22]. These results may indicate that the IRF family plays a key role in the intestinal immune system. The expression levels of IRF5 in the immune and 

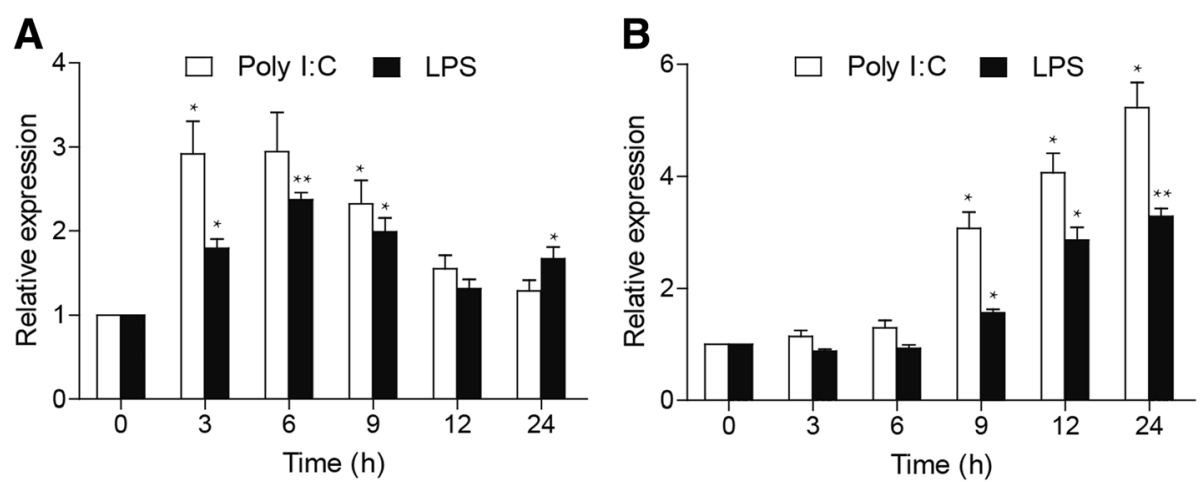

Fig. 7 Expression levels of cCIRF5 in the PBLS (a) and HKLs (b) induced by ploy l:C and LPS in vivo. Total RNA was extracted from control and challenged samples at $0,3,6,9,12$ and $24 \mathrm{hpi}$. The expression was normalized to S11 and shown relative to control. The data are presented as the mean \pm S.D. $(n=3)$. Significant values in comparison to the control are indicated by ${ }^{*} p<0.05$ and ${ }^{*} *<0.01$

non-immune tissues of zebrafish, rock bream and turbot were weakly affected by infectious poly $\mathrm{I}: \mathrm{C}$, which is in accordance with our study $[15,18,36]$. In contrast, the induction magnitude of IRF5 was much stronger in the tissues of poly I:C-injected Japanese flounder [17]. In addition, fish (grass carp, Japanese flounder, rock bream, turbot and half-smooth tongue soles) IRF5 genes were reported to be significantly induced by different pathogens (grass carp reovirus, lymphocystis disease virus, iridovirus, turbot reddish body iridovirus and megalocytivirus) $[14,15,17-19,36]$. These results indicate that fish IRF5 may respond to different pathogens in a tissueor virus-specific manner, but the mechanism involved require further investigation.

TNF $\alpha$, a potent proinflammatory cytokine produced following PAMP recognition by PRRs, and ISGs were also observed at different time points upon the stimulation of poly I:C in vivo by real-time PCR. The highest induction of ccTNF $\alpha$ was detected in the spleen and the liver at 24 hpi (42.3- fold) and $6 \mathrm{hpi}$ (20.4-fold), respectively (Fig. 6). This finding is potentially because the spleen and liver are key innate immune tissues in fish that have a rich resident population of macrophages and lymphocytes, which secrete large quantity of TNF $\alpha$ upon stimulation with pathogens. The Skin, the gills and the intestine (including foregut and hindgut) are important mucosal lymphoid tissues in fish [37]. In this study, following poly I:C challenge, ccISG15 and ccPKR in the foregut and the hindgut were significantly induced. This result indicates that IRF5 might play an important role in mucosal immune system. Further, in our preliminary studies, gene expression of ccIFN and ccMx in all tested tissues was also significantly induced by poly I:C stimulation (unpublished data). In Japanese flounder and turbot, Mx expression was strongly induced by poly I:C in the head kidney and the gills $[38,39]$. Thus, these results suggest a role of ccIRF5 in the activation of downstream antiviral pathways.

In accordance with the in vivo study, ccIRF5 transcripts in common carp PBLs and HKLs were induced upon the stimulation of poly I:C and LPS (Fig. 7). The reason for this phenomenon might because of the
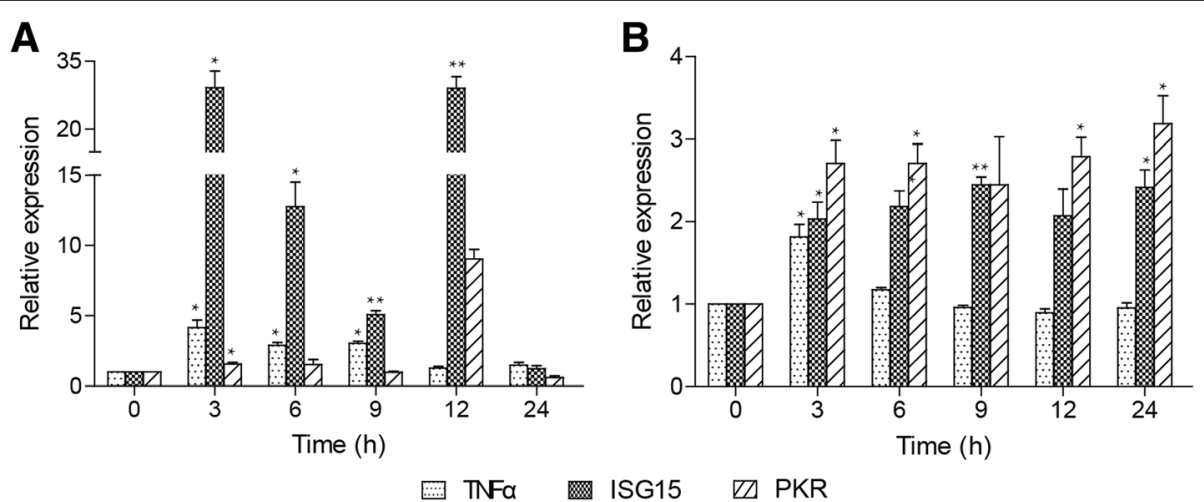

Fig. 8 Expression analysis of cCTNFa and ccISGs in common carp PBLs upon poly I:C (a) and LPS (b) challenges. Total RNA was extracted from the challenged samples at each time point (shown in Fig. 7). The expression was normalized to $\$ 11$ and shown relative to control. The data are presented as the mean \pm S.D. $(n=3)$. Significant values in comparison to the control are indicated by ${ }^{*} p<0.05$ and ${ }^{*} p<0.01$ 
presence of immune cells, including monocytes, granulocytes and lymphocytes [40, 41]. Notably, the highest induction of ccIRF5 in the PBLs (3 to $6 \mathrm{hpi}$ ) was earlier than the induction in the HKLs (24 hpi). This might be due to that IRF family members perform different cellular activities in a cell type-specific way [42].

$\mathrm{Mx}$ and TNF $\alpha$ were induced upon transfection of IRF5 in rock bream heart cells [18]. In this study, ccTNF $\alpha$, ccISG15 and ccPKR transcripts in common carp PBLs were significantly up-regulated upon the two stimuli (Fig. 8). Intriguingly, following LPS stimulation, ccISG15 and cCPKR expression reached the highest level at $24 \mathrm{hpi}$, which was later than the stimulation by poly I:C. And the fold change of the three genes induced by poly I:C (4.1- to 29.1-fold) was stronger than that induced by LPS (1.8- to 3.2fold). This phenomenon may be due to that IRF5 mediates immune response in a pathogen-specific way. In accordance with our study, expression of IRF5 in half-smooth tongue sole was significantly increased post bacterial infection in vivo [19]. Therefore, these results are signifying ccIRF5 may be not only play an the important role in regulating the antiviral immune response, as reported for mammalian IRF5, but also can respond to bacterial challenge $[12,43]$.

\section{Conclusions}

In summary, we report the cloning and characterization of an IRF5 gene from common carp at transcriptional and genomic DNA levels. Furthermore, we describe tissue distribution and in vivo and in vitro induction of ccIRF5, ccTNFo and ccISGs upon stimulation with poly I:C and LPS. Our findings suggest that IRF5 might play an important role in regulating the antiviral and antibacterial response in fish, and these results could provide a clue for preventing common carp infection by pathogenic microorganisms present in the aquatic environment.

\section{Additional file}

Additional file 1: Intron sequences of the cclRF5 gene. Intron sizes are $495,804,225,131,88,761,518$, and 766 bp. (TXT $3 \mathrm{~kb}$ )

\section{Abbreviations}

ANOVA, analysis of variance; $c c$, common carp; DBD, DNA binding domain; $H K L s$, leukocytes from head kidney; hpi, hours post-injection; IAD, interferon regulatory factor-associated domain; ICSAT, interferon consensus sequencebinding protein in adult T-cell leukemia cell line or activated T cells; ICSBP, interferon consensus sequence binding protein; IFN, interferon; IL, interleukin; IRF, interferon regulatory factor; IRF-E, interferon regulatory factor binding element; IRSE, interferon stimulated response element; ISG, interferon stimulated gene; ISGF3ץ, interferon stimulated gene factor 3 gamma; LPS, lipopolysaccharides; LSIRF, Iymphoid-specific interferon regulatory factor; $\mathrm{MR}$, middle region; $\mathrm{NCBI}$, National Center for Biotechnology Information; NLSs, nuclear localization signals; ORF, open reading frame; PBLs, leukocytes from peripheral blood; PBS, phosphatebuffered saline; PIP, PU.1 interaction partner; PKR, protein kinase R; poly I:C, polyinosinic:polycytidylic acid; RACE, rapid amplification of the CDNA ends; RIG-I, retinoic acid-inducible gene I; SMART, simple modular architecture research tool; SVCV, spring viraemia of carp virus; TNFa, tumour-necrosis factor a; UTR, untranslated region; $V A D$, virus activated domain

\section{Acknowledgements}

Not applicable.

\section{Funding}

This research was supported by the Shandong Provincial Natural Foundation of China (ZR2014CZ004 and ZR2014CQ051) and the University Independent Innovation Project of Jinan (200906020).

\section{Availability of data and materials}

The datasets supporting the conclusions of this article is available in the GenBank (Accession Number: KP979609) and TreeBase repository (http:// purl.org/phylo/treebase/phylows/study/TB2:S19362).

\section{Authors' contributions}

YYZ and LGA participated in the design of the study, performed the experiments, collected and analysed data, and drafted the manuscript. CCQ cloned and sequenced the cCIRF5 gene. SJS and HL helped with the animal experiments. FMZ participated in the acquisition of preliminary data and performed the statistical analysis. GWY conceived the study and participated in its design and coordination. All authors read and approved the final manuscript.

\section{Competing interests}

The authors declare that they have no competing interests.

\section{Consent for publication}

Not applicable.

\section{Ethics approval and consent to participate}

The protocol was approved by the Ethics Committee on Animal Experiments of the Medical School of Shandong University (Permit Number: ECAESDUSM 20123009).

Received: 13 January 2016 Accepted: 16 June 2016 Published online: 27 June 2016

\section{References}

1. Nguyen $\mathrm{H}$, Hiscott J, Pitha PM. The growing family of interferon regulatory factors. Cytokine Growth Factor Rev. 1997;8(4):293-312.

2. Harada H, Taniguchi T, Tanaka N. The role of interferon regulatory factors in the interferon system and cell growth control. Biochimie. 1998:80(8-9):641-50.

3. Barnes B, Lubyova B, Pitha PM. On the role of IRF in host defense. J Interferon Cytokine Res. 2002;22(1):59-71.

4. Mamane Y, Heylbroeck C, Genin P, Algarte M, Servant MJ, LePage C, et al. Interferon regulatory factors: the next generation. Gene. 1999;237(1):1-14.

5. Huang B, Qi ZT, Xu Z, Nie P. Global characterization of interferon regulatory factor (IRF) genes in vertebrates: glimpse of the diversification in evolution. BMC Immunol. 2010;11:22

6. Paun A, Pitha PM. The IRF family, revisited. Biochimie. 2007;89(6-7):744-53.

7. Eroshkin A, Mushegian A. Conserved transactivation domain shared by interferon regulatory factors and Smad morphogens. J Mol Med (Berl). 1999;77(5):403-5.

8. Barnes BJ, Moore PA, Pitha PM. Virus-specific activation of a novel interferon regulatory factor, IRF-5, results in the induction of distinct interferon alpha genes. J Biol Chem. 2001;276(26):23382-90.

9. Tamura T, Yanai H, Savitsky D, Taniguchi T. The IRF family transcription factors in immunity and oncogenesis. Annu Rev Immunol. 2008;26:535-84.

10. Honda K, Taniguchi T. IRFs: master regulators of signalling by Toll-like receptors and cytosolic pattern-recognition receptors. Nat Rev Immunol. 2006;6(9):644-58.

11. Barnes BJ, Kellum MJ, Field AE, Pitha PM. Multiple regulatory domains of IRF-5 control activation, cellular localization, and induction of chemokines that mediate recruitment of T lymphocytes. Mol Cell Biol. 2002;22(16):5721-40.

12. Paun A, Reinert JT, Jiang Z, Medin C, Balkhi MY, Fitzgerald KA, et al. Functional characterization of murine interferon regulatory factor 5 (IRF-5) 
and its role in the innate antiviral response. J Biol Chem. 2008;283(21): 14295-308.

13. Takaoka A, Yanai H, Kondo S, Duncan G, Negishi H, Mizutani T, et al. Integral role of IRF-5 in the gene induction programme activated by Toll-like receptors. Nature. 2005;434(7030):243-9.

14. Xu QQ, Chang MX, Xiao FS, Huang B, Nie P. The gene and virus-induced expression of IRF-5 in grass carp Ctenopharyngodon idella. Vet Immunol Immunopathol. 2010;134(3-4):269-78.

15. Xiang Z, Dong C, Qi L, Chen W, Huang L, Li Z, et al. Characteristics of the interferon regulatory factor pairs zfIRF5/7 and their stimulation expression by ISKNV Infection in zebrafish (Danio rerio). Dev Comp Immunol. 2010; 34(12):1263-73.

16. Leong JS, Jantzen SG, von Schalburg KR, Cooper GA, Messmer AM, Liao NY, et al. Salmo salar and Esox lucius full-length CDNA sequences reveal changes in evolutionary pressures on a post-tetraploidization genome. BMC Genomics. 2010;11:279.

17. Hu GB, Lou HM, Dong XZ, Liu QM, Zhang SC. Characteristics of the interferon regulatory factor 5 (IRF5) and its expression in response to LCDV and poly I:C challenges in Japanese flounder, Paralichthys olivaceus. Dev Comp Immunol. 2012;38(2):377-82.

18. Wickramaarachchi WD, Wan Q, Lim BS, Jung HB, De Zoysa M, Park MA, et al. Genomic characterization of interferon regulatory factor 5 from rock bream (Oplegnathus fasciatus) and its role in antiviral defense. Fish Shellfish Immunol. 2014;37(2):256-67.

19. Zhang J, Li YX, Hu YH. Molecular characterization and expression analysis of eleven interferon regulatory factors in half-smooth tongue sole, Cynoglossus semilaevis. Fish Shellfish Immunol. 2015;44(1):272-82.

20. Liu S, Zhang Y, Zhou Z, Waldbieser G, Sun F, Lu J, et al. Efficient assembly and annotation of the transcriptome of catfish by RNA-Seq analysis of a doubled haploid homozygote. BMC Genomics. 2012;13:595.

21. Xiaoni G, Zhuo C, Xuzhen W, Dengqiang W, Xinwen C. Molecular cloning and characterization of interferon regulatory factor 1 (IRF-1), IRF-2 and IRF-5 in the chondrostean paddlefish Polyodon spathula and their phylogenetic importance in the Osteichthyes. Dev Comp Immunol. 2012;36(1):74-84.

22. Feng $H$, Liu $H$, Kong $R$, Wang L, Wang $Y, H u$ W, et al. Expression profiles of carp IRF-3/-7 correlate with the up-regulation of RIG-I/MAVS/TRAF3/TBK1, four pivotal molecules in RIG-I signaling pathway. Fish Shellfish Immunol. 2011:30(4-5):1159-69.

23. Fournier G, Boutier M, Stalin Raj V, Mast J, Parmentier E, Vanderwalle $P$, et al. Feeding Cyprinus carpio with infectious materials mediates cyprinid herpesvirus 3 entry through infection of pharyngeal periodontal mucosa. Vet Res. 2012:43:6.

24. NCBI [http://www.ncbi.nlm.nih.gov/blast].

25. SMART [http://smart.embl-heidelberg.de/].

26. Rymuszka A, Sieroslawska A. Effects of neurotoxin - anatoxin-a on common carp (Cyprinus carpio L.) innate immune cells in vitro. Neuro endocrinology letters. 2011;32 Suppl 1:84-8.

27. Engelsma MY, Stet RJ, Schipper H, Verburg-van Kemenade BM. Regulation of interleukin 1 beta RNA expression in the common carp, Cyprinus carpio L. Dev Comp Immunol. 2001;25(3):195-203.

28. Escalante CR, Yie J, Thanos D, Aggarwal AK. Structure of IRF-1 with bound DNA reveals determinants of interferon regulation. Nature. 1998; 391(6662):103-6.

29. Lin R, Mamane $Y$, Hiscott J. Structural and functional analysis of interferon regulatory factor 3: localization of the transactivation and autoinhibitory domains. Mol Cell Biol. 1999;19(4):2465-74.

30. Chen W, Lam SS, Srinath H, Jiang Z, Correia JJ, Schiffer CA, et al. Insights into interferon regulatory factor activation from the crystal structure of dimeric IRF5. Nat Struct Mol Biol. 2008;15(11):1213-20.

31. Yabu T, Hirose H, Hirono I, Katagiri T, Aoki T, Yamamoto E. Molecular cloning of a novel interferon regulatory factor in Japanese flounder, Paralichthys olivaceus. Mol Mar Biol Biotechnol. 1998;7(2):138-44.

32. Richardson MP, Tay BH, Goh BY, Venkatesh B, Brenner S. Molecular cloning and genomic structure of a gene encoding interferon regulatory factor in the pufferfish (Fugu rubripes). Mar Biotechnol (NY). 2001;3(2):145-51.

33. Sun $B$, Chang $M$, Chen D, Nie P. Gene structure and transcription of IRF-2 in the mandarin fish Siniperca chuatsi with the finding of alternative transcripts and microsatellite in the coding region. Immunogenetics. 2006;58(9):774-84.
34. Shi Y, Zhang YB, Zhao Z, Jiang J, Zhang QY, Gui JF. Molecular characterization and subcellular localization of Carassius auratus interferon regulatory factor-1. Dev Comp Immunol. 2008;32(2):134-46.

35. Holland JW, Karim A, Wang T, Alnabulsi A, Scott J, Collet B, et al. Molecular cloning and characterization of interferon regulatory factors 4 and 8 (IRF-4 and IRF-8) in rainbow trout, Oncorhynchus mykiss. Fish Shellfish Immunol. 2010;29(1):157-66.

36. Xia J, Hu GB, Dong XZ, Liu QM, Zhang SC. Molecular characterization and expression analysis of interferon regulatory factor 5 (IRF-5) in turbot, Scophthalmus maximus. Fish Shellfish Immunol. 2012;32(1):211-8.

37. Magnadottir B. Innate immunity of fish (overview). Fish Shellfish Immunol. 2006;20(2):137-51.

38. Hu G, Yin X, Xia J, Dong X, Zhang J, Liu Q. Molecular cloning and characterization of interferon regulatory factor 7 (IRF-7) in Japanese flounder, Paralichthys olivaceus. Fish Shellfish Immunol. 2010;29(6):963-71.

39. Hu GB, Xia J, Lou HM, Chen XL, Li J, Liu QM. An IRF-3 homolog that is upregulated by DNA virus and poly I:C in turbot, Scophthalmus maximus. Fish Shellfish Immunol. 2011;31(6):1224-31.

40. Neumann NF, Stafford JL, Barreda D, Ainsworth AJ, Belosevic M. Antimicrobial mechanisms of fish phagocytes and their role in host defense. Dev Comp Immunol. 2001;25(8-9):807-25.

41. Li J, Barreda DR, Zhang YA, Boshra H, Gelman AE, Lapatra S, et al. B lymphocytes from early vertebrates have potent phagocytic and microbicidal abilities. Nat Immunol. 2006;7(10):1116-24.

42. Hu G, Barnes BJ. IRF-5 is a mediator of the death receptor-induced apoptotic signaling pathway. J Biol Chem. 2009;284(5):2767-77.

43. Barnes BJ, Kellum MJ, Pinder KE, Frisancho JA, Pitha PM. Interferon regulatory factor 5 , a novel mediator of cell cycle arrest and cell death. Cancer Res. 2003;63(19):6424-31.

\section{Submit your next manuscript to BioMed Central and we will help you at every step:}

- We accept pre-submission inquiries

- Our selector tool helps you to find the most relevant journal

- We provide round the clock customer support

- Convenient online submission

- Thorough peer review

- Inclusion in PubMed and all major indexing services

- Maximum visibility for your research

Submit your manuscript at www.biomedcentral.com/submit 